\title{
Planejamento familiar na perspectiva do desenvolvimento
}

\author{
Family planning under the view of development
}

Júlio César dos Santos ${ }^{1}$

Patrícia Martins de Freitas ${ }^{1}$

${ }^{1}$ Grupo de Pesquisa Saúde,

Educação e

Desenvolvimento, Centro

de Ciências da Saúde,

Universidade Federal do

Recôncavo da Bahia. Av.

Carlos Amaral 1.015,

Bairro Cajueiro, Campus

Universitário. 44570-000

Santo Antônio de Jesus BA.

economiapsicologia@gmail.com

\begin{abstract}
The present essay has as objective to critically analyze the current limitations of the family planning applied in public health and to consider changes considering the model of social sciences applied to health and the perspective of the family development. The proposal of this work appeared because of the high number of women who requested maternity assistance in the region of the Recôncavo of Bahia in the year of 2006. By analyzing the variables and methods involved in the family planning, it was possible to verify the following fault: responsibility and exclusive participation of the women, focus in the individual, the number of children as main goal of the planning, biological model, disrespect of the cultural aspects of the partner. In this essay we present the proposal of family planning of the perspective of the development that will be tested as a model of intervention in a future study.
\end{abstract}

Key words Family planning, Socioeconomic variable, Familiar development
Resumo O presente ensaio tem como objetivo analisar criticamente as limitações atuais do planejamento familiar aplicado na saúde pública $e$ propor mudanças considerando o modelo das ciências sociais aplicadas à saúde e a perspectiva do desenvolvimento familiar. A proposta deste trabatho surgiu do elevado número de mulheres que solicitara o auxílio maternidade na região do Recôncavo da Bahia no ano de 2006. Através da análise das variáveis e dos métodos envolvidos no planejamento familiar, foi possível verificar as seguintes falhas: responsabilidade e participação exclusiva das mulheres, foco no indivíduo, o número de filhos como principal meta do planejamento, modelo biológico, desconsideração dos aspectos socioculturais. Neste ensaio, apresentamos a proposta de planejamento familiar na perspectiva do desenvolvimento, que será testada como modelo de intervenção em um estudo futuro.

Palavras-chave Planejamento familiar, Variáveis socioeconômicas, Desenvolvimento familiar 


\section{Introdução}

O estudo do planejamento familiar tem sido tema de uma ampla discussão que envolve diversas áreas, especialmente a política, a economia e a psicossocial. Atualmente, as discussões sobre a temática são bastante cautelosas, retirando o foco da dicotomia controle versus não controle, que é reconhecida como desgastada ${ }^{1}$. O planejamento familiar é atualmente foco de um dos principais programas na área da saúde, o Programa de Saúde da Família (PSF), e as limitações de sua aplicação podem ter consequências importantes para o desenvolvimento familiar ${ }^{2,3}$.

A criação do PSF e a do planejamento familiar como parte de suas ações demonstram que a saúde da família é umas das prioridades para a saúde pública, o que revela ser um indicativo de que o governo compreende a importância da família como sistema (ver Agenda Nacional de Prioridades de Pesquisa em Saúde, 2005) ${ }^{4}$. A ação sobre a unidade familiar é maior do que a ação isolada com os membros da família ${ }^{5}$. Apesar do foco sobre a família nas políticas de saúde pública, algumas lacunas têm sido observadas. Segundo Carvalho e Brito ${ }^{1}$, o planejamento familiar não atende às demandas de mulheres pobres. Dessa forma, o planejamento familiar apresenta restrições quando aplicado a mulheres que pertencem a classes sociais menos favorecidas ${ }^{6}$.

As causas para as restrições nas ações do PSF são discutidas por diferentes pesquisadores, e as hipóteses mais frequentemente convergentes são problemas com o processo informativo e pouco acesso aos métodos anticoncepcionais ${ }^{2,7-9}$. Segundo Camiá et al. ${ }^{10}$, a qualidade da orientação é fundamental para obtenção de resultados positivos do Programa. Nesse caso, a eficiência do processo educativo aumenta as possibilidades de efetividade do planejamento familiar, ou seja, capacita os usuários na tomada de decisões sobre a arquitetura e a dinâmica da família. A qualidade da informação é também discutida por Bruce ${ }^{8}$. Segundo a autora, é necessário que o usuário compreenda e retenha a informação, para que seja efetivo o uso dos métodos anticoncepcionais.

Apesar de considerar a importância do processo educativo e dos trabalhos já desenvolvidos nas áreas do planejamento familiar, muitos problemas ainda são muito expressivos. Considerando essa expressividade, o presente artigo foi desenvolvido com o objetivo de aprofundar as hipóteses explicativas sobre as falhas do programa de planejamento familiar, bem como de apresentar propostas que possam aumentar a eficá- cia do programa nas classes socioeconômicas mais baixas.

A realização deste estudo é decorrente de uma análise baseada nos dados populacionais e das estatísticas do Instituto Nacional do Seguro Social (INSS) na região do Recôncavo Baiano. A Secretaria Municipal de Saúde de Santo Antônio de Jesus (BA), cidade polo da microrregião na saúde, juntamente com o Ministério Público Estadual e a gerência executiva do INSS na cidade, a qual é formada por 11 agências que atendem 43 municípios, identifica um aumento significativo na solicitação de auxílio-maternidade pelas mulheres da zona rural da região.

A presença da Universidade Federal do Recôncavo da Bahia permitiu que essa demanda social se constituísse numa questão de pesquisa, oferecendo possíveis mecanismos para minimizar o problema. Por meio da revisão da literatura, foi possível verificar que o planejamento familiar tem um foco específico no controle da natalidade e, mais recentemente, na saúde da mulher e no uso de métodos anticoncepcionais. A análise do modelo de planejamento familiar e suas limitações contribuiu para a formulação de hipóteses explicativas para os problemas tão frequentes do planejamento familiar, bem como para a proposição de mudanças para ações de planejamento familiar.

Para compreendermos a relação entre os dados observados, as limitações identificadas e as mudanças propostas, a análise será estruturada em tópicos que contemplam os aspectos históricos, conceituais e característicos do planejamento familiar; as principais falhas e hipóteses explicativas; as mudanças propostas e os resultados esperados.

\section{Os aspectos históricos, conceituais e característicos do planejamento familiar}

O planejamento familiar surge imerso nas contradições teórico-ideológicas associadas à crise do crescimento populacional. Nesse período, os estudiosos da demografia ${ }^{11,12}$ previam impactos expressivos no desenvolvimento socioeconômico dos países subdesenvolvidos, como eram considerados na época. O aumento indiscriminado da população estava diretamente relacionado ao aumento da pobreza e das consequências desta ${ }^{1,7}$.

Os dados atuais das taxas de fecundidades sugerem que o Brasil se caracteriza por uma sociedade com baixa fecundidade. Entretanto, esses dados devem ser analisados por região. As 
reduzidas taxas de fecundidade das regiões mais desenvolvidas do país mascaram os problemas presentes em regiões como o Nordeste brasileiro. A comparação das taxas de fecundidade exemplifica as controvérsias regionais presentes na sociedade brasileira.

A taxa de fecundidade brasileira em 2006 se reduziu para dois filhos por mulher e já é uma das menores da América Latina, próxima à de países desenvolvidos como França $(1,9)$ e Suécia $(1,7)$, e igual à dos Estados Unidos. Mas se forem comparadas regiões, classe social e grau de estudo, os dados da Pesquisa Nacional por Amostra de Domicílio (PNAD) 2006, divulgada pelo Instituto Brasileiro de Geografia e Estatística (IBGE), apontam que as mães pobres têm o dobro - ou mais - de filhos que as de classe média ou alta. $\mathrm{O}$ IBGE demonstra uma queda mais significativa nas regiões Sul e Sudeste e menor no Nordeste e no Centro-Oeste do país ${ }^{1,12}$.

A comparação entre as taxas de fecundidade foi baseada em dados da Organização Mundial da Saúde (OMS) e da PNAD 2005. De acordo com o IBGE, a taxa de fecundidade em 2005 era de 2,1 filhos por mulher, e o número vem declinando desde os anos 60, quando as brasileiras tinham em média seis filhos - na década de 80 , a média já era de quatro filhos.

Esses dados são reflexos dos problemas identificados ainda na década de 70, quando o Sul e o Sudeste demonstravam significativo declínio na taxa de fecundidade, enquanto as regiões Norte e Nordeste mantinham taxas elevadas ${ }^{11}$. A queda na taxa de fecundidade foi mais acentuada nas regiões mais desenvolvidas do país. A relação entre status socioeconômico e efetividade do planejamento familiar tem sido evidenciada por diferentes estudos $2,7,11-14$.

O planejamento familiar foi construído historicamente como aplicação de métodos para controlar o número de filhos por famílias ${ }^{1,11,15}$. Entretanto, quais os critérios utilizados por um casal para definir o número de filhos? Alguns estudos evidenciam que o critério econômico tem sido bastante utilizado ${ }^{11,14}$. As famílias avaliam a renda e tentam "intuir" o número de filhos compatível. É possível perceber que o princípio da sustentabilidade familiar está presente. A adequação do número de filhos à renda familiar representa a viabilidade dos recursos básicos para o desenvolvimento de seus membros ${ }^{16}$. No entanto, o programa de planejamento familiar não oferece orientações para que as famílias analisem a sustentabilidade ${ }^{16}$.

O planejamento familiar foi definido como instrumento da assistência materno-infantil e ad- vém de um processo de informação e de educação aos casais e à população em geral, sobre a reprodução, a importância da família na comunidade, o papel da mulher, o papel do pai e do filho dentro desse contexto e, finalmente, sobre as repercussões de tudo isso na comunidade ${ }^{11}$.

Atualmente, Camiá et al. ${ }^{10}$ definem o planejamento familiar como um mecanismo importante na prevenção primária de saúde, auxiliando as usuárias com informações necessárias para a escolha e para o uso efetivo de métodos anticoncepcionais mais adequados. Apesar de esta definição contemplar os elementos de prevenção à saúde, ainda é uma visão focada apenas na saúde da mulher e no controle do número de filhos. No estudo de Osis et $a l^{7}$, a análise da participação masculina nas atividades de planejamento familiar revelou envolvimento bastante reduzido dos homens e a inexistência de programas para saúde sexual e reprodutiva masculina em todas as capitais.

Os serviços de planejamento familiar tradicionais atuam em função da vida reprodutiva com responsabilidade exclusivamente feminina e prioridade social. As opções contraceptivas mais utilizadas pelas mulheres são sugeridas pelos serviços de saúde e requerem desenvolvimento tecnológico e monitoramento médico, consolidando o processo de medicalização da população. Na região do Recôncavo Baiano, observa-se que há respostas diferentes entre as populações rurais e urbanas às campanhas publicitárias de planejamento familiar comunicadas ao grande público. Tais opções contraceptivas são indicadas genericamente e não são necessariamente adequadas à concepção reprodutiva da população rural, dados que desconsideram sua diversidade socioeconômica como anos de escolaridade, crenças, determinantes da saúde e as tradições locais.

O planejamento familiar como mecanismo de limitação da reprodução feminina é composto de: visita das mulheres ao ginecologista, participação das reuniões de planejamento familiar e compra do contraceptivo ou "ganho" do Posto de Saúde. Contudo, as mulheres do meio urbano não assistem a todas as reuniões de orientação do planejamento familiar; o comportamento das mulheres da zona rural é semelhante. A presença nas reuniões tem como objetivo obter o contraceptivo. Outro aspecto importante é a centralização do planejamento familiar na figura feminina. Na maioria das vezes, a mulher participa sozinha das reuniões de orientação para o planejamento familiar. A mulher faz a escolha do contraceptivo e assume a responsabilidade pela decisão da quantidade de filhos. 
A existência de um benefício social específico para as mulheres gestantes da zona rural pode estar funcionando como potencializador das falhas do planejamento familiar para essa população especificamente. $\mathrm{O}$ auxílio-maternidade tem como função garantir a renda durante o período de licença, sendo sua duração de quatro meses. Com esse benefício, a família pode obter recursos para auxiliar na fase inicial de desenvolvimento da prole.

Contudo, o aumento no número de solicitações desse benefício demonstra que existem distorções de sua finalidade. No Recôncavo Baiano, por exemplo, o auxílio-maternidade é o segundo benefício mais pago pela Previdência, perdendo somente para o auxílio-doença. Segundo dados do INSS, em 2006, na agência do INSS de Santo Antônio de Jesus, 4.800 mulheres que moram no campo receberam o salário-maternidade. $\mathrm{O}$ benefício pode ser requerido a partir do oitavo mês de gestação até o quinto ano de vida da criança. Para isso, a mulher precisa comprovar que mora e trabalha na zona rural.

As consequências demográficas do auxíliomaternidade rural no município de Santo Antônio de Jesus sugerem que as mulheres têm filhos para poder receber o benefício, o que acaba sendo o sustento da família. A relação entre a renda oferecida pelo auxílio-maternidade e o aumento no número de filhos por mulher na zona rural é um indicativo da ineficiência do programa de planejamento. $\mathrm{O}$ aspecto socioeconômico presente no planejamento familiar é evidenciado diante dos impactos provocados na seguridade social. A reação em cadeia provocada pelo aumento da taxa de fecundidade das mulheres na zona rural do Recôncavo da Bahia inicia-se com a Previdência, mas, em poucos anos, poderá afetar também os serviços públicos de saúde, de educação e de segurança. Esse fenômeno indica que o planejamento familiar não é uma questão do ponto de vista apenas biológico, mas também social ${ }^{17}$.

$\mathrm{O}$ aumento no número de beneficiárias rurais do auxílio-maternidade é resultado das falhas do modelo linear e tradicional de planejamento familiar direcionado para a fertilidade e a fecundidade feminina. Alguns estudos, em especial da área da saúde, conjugam essa definição com metodologias contraceptivas, o que poderá ser ampliado com a contribuição com outras ciências como as sociais aplicadas.

A aplicação de um planejamento familiar multidisciplinar envolvendo a área de ciências sociais aplicadas pode trazer um novo modelo para se lidar com esse fenômeno. A elaboração de um novo modelo de intervenção para o planejamento familiar deve resultar inicialmente $\mathrm{da}$ análise das principais falhas dos programas de planejamento familiar utilizados atualmente.

\section{A perspectiva do desenvolvimento familiar}

O sistema familiar, definido como um grupo de pessoas que compartilham o seu dia a dia, desempenha uma importante função no desenvolvimento e na promoção da qualidade de vida e do bem-estar de seus membros ${ }^{18,19}$.

O ciclo de vida de uma família deve considerar os termos desenvolvimentais e do relacionamento intergeracional. Segundo Duval ${ }^{20}$, o ciclo de vida pode ser dividido em oito estágios referentes aos caminhos percorridos pelos membros da família, como casamento, nascimento e educação dos filhos, saída dos filhos do lar, aposentadoria e morte. Percebe-se também que, em cada estágio do ciclo de vida, existe um complexo de papéis distintos para os membros da família, uns em relação aos outros. Duval ${ }^{20}$ focaliza a educação e o desenvolvimento dos filhos como o elemento organizador da vida familiar.

O contexto social, econômico e político, com seu impacto sobre as famílias movendo-se através de diferentes fases do ciclo de vida, em cada momento na história, não pode ser ignorado. Os modelos de planejamento familiar típicos deixam de considerar os efeitos significativos da etnicidade, da religião, das classes sociais, em todos os aspectos de quando e de que maneira uma família faz suas transições de uma fase para outra. As famílias possuem rituais, valores, uma dinâmica sistêmica, que interferem nas diferentes transições, de acordo com o background cultural da família ${ }^{21}$.

O fluxo de ansiedade numa família ocorre horizontal e verticalmente. O último ocorre em um sistema que inclui padrões de relacionamento e funcionamento transmitidos para as gerações seguintes de uma família através da triangulação emocional. O fluxo horizontal no sistema inclui a ansiedade produzida pelos estresses na família conforme ela avança no tempo, lidando com as mudanças e com as transições do ciclo de vida familiar. Neste modelo, quando o estresse horizontal (desenvolvimental) faz uma interseção com o vertical (transgeracional), existe um aumento importante da ansiedade no sistema ${ }^{22}$.

Os elementos sociais como as desigualdades na distribuição de renda podem ser variáveis es- 
tressoras do sistema. Quando em contato com uma fase de transição, aumentam a tensão e a instabilidade do sistema. As desigualdades de renda e política têm aumentado em nossa cultura, e as diferenças entre famílias são discrepantes. Uma proposição clássica é a diferença 10/90: enquanto $10 \%$ da população possuem $90 \%$ da renda, $90 \%$ da população possuem apenas $10 \%$ da ren$\mathrm{da}^{16}$. Os indicativos socioeconômicos destacam a importância de se considerarem tais variáveis nos trabalhos voltados para as famílias. As políticas públicas destinadas ao planejamento familiar não lidam com as diferenças socioeconômicas e geográficas, o que torna o modelo frágil para famílias pobres, especialmente as rurais ${ }^{16}$.

Hines $^{23}$ estudou as famílias negras pobres de hereditariedade afro-americanas. Nesse estudo, foi considerado que as famílias não podem esperar nada além de um trabalho esporádico, inferior. $\mathrm{O}$ prejuízo no ciclo de vida delas é marcado pelo desemprego, pela desnutrição, pelos nascimentos préconjugais, pela instabilidade e violência intrafamiliar, pelos distúrbios mentais, pela delinquência, pelo abuso de substâncias, por um alto índice de mortalidade infantil, pela incapacidade física, pela morte precoce e pelos estresses contínuos de habitações inadequadas e constantes dívidas ${ }^{23}$.

O ciclo de vida de famílias de baixa renda é marcado por inúmeras crises, e suas capacidades adaptativas são frequentemente levadas além de limites humanos. Os prejuízos para o desenvolvimento envolvem tanto os fatores socioeconômicos quanto os psicossociais. Outra característica é o empobrecimento emocional que também é parte desse perfil. Alguns, inseridos num contexto de crônico desemprego e discriminação, parecem ficar extremamente limitados em sua capacidade de funcionar a uma maneira que permita aos membros da família progredirem ${ }^{24}$.

No entanto, ser pobre não significa automaticamente ser uma família disfuncional. Existem famílias com recursos inadequados, mas que conseguem satisfazer as necessidades desenvolvimentais básicas de seus membros. A pobreza passa a ser um problema quando existem aspectos sociais que servem para mantê-la numa posição de miséria e de impotência ${ }^{25}$; então a pobreza possui um desenvolvimento circular. $\mathrm{O}$ desemprego tecnológico, ligado ao novo paradigma técnico-econômico, é um exemplo de perpetuação da pobreza. Na medida em que maior instrução e habilidade técnica tornaram-se críticas, esse grupo geralmente não tem a oportunidade de adquiri-la. Eles são os últimos a serem empregados e os primeiros a serem despedidos.
Nesse caso, a autoestima está sensível com a ausência de empregos adequados, implicando sentimentos de impotência, raiva e desespero.

Hines $^{23}$ aponta ainda que as famílias negras pobres são: (1) frequentemente chefiadas por mulheres e do tipo de família ampliada (constituída por membros da família nuclear e da família de origem - exemplo: a presença de tios, avós, primos); (2) e contam com poucos recursos para ajudá-las a lidar com esses fatores estressores, além de sua dependência do segundo e do terceiro setores econômicos, como já explicitado.

Considerando as características das famílias do estudo de Hines ${ }^{23}$, os trabalhos desenvolvidos com as famílias na área da saúde têm a obrigatoriedade de lidar com as variáveis socioeconômicas e psicossociais. O modelo de planejamento familiar atual utilizado pela saúde pública no Brasil é baseado na saúde reprodutiva da mulher. Todavia, a família é compreendida como um sistema em que o indivíduo não deve ser tratado separadamente, mas fazendo parte de uma rede familiar que possui regras implícitas e explícitas ${ }^{26}$.

\section{As fragilidades do programa de planejamento familiar}

Os programas desenvolvidos por ações governamentais são caracterizados por responder às necessidades de saúde pública. No caso do planejamento familiar, os problemas de saúde pública em questão são o controle da natalidade, a redução do aborto, a saúde da mulher, a redução da pobreza etc. O controle do número de filhos foi uma das primeiras metas do planejamento familiar no Brasil e tinha como objetivo evitar altas taxas de fecundidade. Os problemas como a mortalidade infantil, aborto, famílias numerosas e consequentemente mais pobres demandavam uma política de saúde ${ }^{27}$.

O foco do planejamento familiar passou a contemplar elementos da saúde da mulher, mas ainda há o foco principal no controle da natalidade. Ainda assim, o planejamento familiar, muitas vezes, só é iniciado depois do primeiro filho. Outra fragilidade é a falta de adequação do programa às características individuais e familiares dos envolvidos. Os valores, as características de personalidade, a história de vida das famílias de origem são elementos que interferem diretamente na estrutura familiar que está sendo formada. Desconsiderar isso é adotar um modelo verticalizado de planejamento familiar, de modo que os envolvidos sejam passivos no processo. 
A análise dos aspectos operacionais envolvidos no planejamento familiar permite observar as possíveis dificuldades de profissionais preparados para informar e orientar sobre os métodos contraceptivos. A limitação para atuar de forma pedagógica é consequência da formação centrada no modelo biomédico ${ }^{2}$. O processo educativo em saúde exige profissionais com conhecimento técnico associado à capacidade de aplicar diferentes metodologias de ensino-aprendizagem. Os métodos utilizados, tais como palestras em grupos ou individuais, podem ser falhos quando reproduzem a ação assistencial característica da saúde, não conseguindo tornar os usuários ativos no processo de planejar sua família.

\section{Inovações no planejamento familiar}

Após a análise das lacunas do atual modelo de planejamento familiar, consideram-se os seguintes principais eixos de ação: foco exclusivo na saúde da mulher; sustentabilidade intuitiva, que se refere à capacidade da família de relacionar os recursos que possui e o número ideal de filhos; desconsideração das características da unidade familiar; processo passivo e com método de ensino-aprendizagem frágil. Para lidar com tais fragilidades, é necessária uma reestruturação da atuação do planejamento familiar.

O planejamento familiar é um método de prevenção e de intervenção na saúde da família, portanto deve considerar a unidade familiar e não apenas a mulher. A fase do ciclo de vida da família deve ser avaliada, bem como suas crenças, valores e tradições. O planejamento deve ser conduzido na forma de programa, passo a passo, com tarefas para tornar o processo ativo para os usuários.

O modelo de planejamento familiar aplicado na saúde pública não realiza avaliações das potencialidades e dos recursos do núcleo familiar. Além disso, não existe nenhuma orientação sobre como relacionar a renda com o número de filhos, como também sobre o investimento de recursos na educação e na saúde a cada novo membro da família. Contudo, estudos realizados por $\operatorname{Sen}^{16}$ demonstram que o trabalho, a alfabetização e a educação das mulheres tendem a reduzir não só as taxas de mortalidade das crianças como também as de natalidade e de fecundidade.

As características aqui referenciadas tiram as mulheres da condição passiva e pouco reconhecida quando exercem o papel de agente. Nesse sentido, o planejamento familiar pode contribuir com o equilíbrio entre as necessidades e desenvolver características mais ativas em mulheres a respeito do planejamento familiar, como tradicionalmente são encontradas nos homens. A avaliação inicial do modelo de planejamento familiar demonstra a importância de reestruturar essa proposta. O planejamento familiar deve estar fundamentado na perspectiva do desenvolvimento, permitindo que a família faça sua própria avaliação, elabore seu plano e gerencie de forma sustentável o crescimento dos membros.

O que deve ser planejado? Até o momento, o planejamento familiar está restrito à escolha do número de filhos, quando tê-los e como evitálos. A relação entre número de filhos e a renda é feita baseada nas questões mais imediatas. Outras questões precisam estar presentes no planejamento familiar; dentre elas, o planejamento dos cuidados com a saúde, do orçamento, da educação, dos aspectos afetivos, direcionados para o desenvolvimento da família. Os membros precisam conhecer mecanismos de estimar a relação entre a renda e o número de filhos. Devem considerar, nesse caso, as necessidades de cada membro em diferentes fases do ciclo de vida individual e familiar.

O impacto de intervenções bem-sucedidas nas famílias poderá contribuir significativamente para o desenvolvimento social, diminuindo a pobreza e as desigualdades sociais por meio da família empreendedora. Ou seja, a família será um empreendimento de seus membros, e os filhos são investimentos de longo prazo. Essa analogia é uma tentativa de alertar o trabalho desenvolvido com as famílias, especialmente as famílias de baixa renda e da zona rural. Estas representam a principal parcela da população que não apresenta resultados efetivos com o planejamento familiar utilizado na saúde pública.

\section{Resultados esperados a partir do planejamento familiar na perspectiva do desenvolvimento}

Através do modelo de planejamento familiar proposto, espera-se contribuir para a promoção da saúde de famílias de baixa renda e provocar mudanças sociais e econômicas. A análise de diferentes estudos demonstra o equilíbrio entre a renda e o número de filhos como um fator positivo para o desenvolvimento social e a diminuição da pobreza.

O planejamento familiar, na perspectiva do desenvolvimento, acrescenta variáveis organiza- 
doras do sistema, permitindo um planejamento baseado na sustentabilidade e no ciclo de vida da família. Para a região do Recôncavo da Bahia, os resultados esperados são voltados para as famílias da zona rural. Como apresentado no presente estudo, as falhas do programa de planejamento familiar para essa população demonstram consequências de alto impacto para a população como um todo.

Outro fator de destaque é o uso dos recursos do auxílio-maternidade, demonstrando que tais famílias lidam com os recursos financeiros avaliando apenas os elementos imediatistas, prejudicando o desenvolvimento sustentável da família. Através desse programa, espera-se uma redução da taxa de fecundidade, associada essa redução com mecanismos de desenvolvimento para as famílias da zona rural em Santo Antônio de Jesus.

O fator educacional parece ser uma variável mediadora da efetividade do planejamento familiar. Portanto, uma das principais mudanças sugeridas por este estudo é a da metodologia utilizada pelos profissionais de saúde; o uso de técnicas psicoeducativas com o objetivo de mudar o comportamento dos indivíduos em relação ao planejamento familiar. Dessa forma, o planejamento familiar torna-se um instrumento de análise e de desenvolvimento para os próprios membros da família.

Outro enfoque que deveria ser contemplado pelo programa de planejamento familiar, mas as pesquisas demonstram estar enfraquecido, é a promoção da saúde e o aumento da qualidade de vida. O foco do planejamento familiar atualmente tem sido o controle do número de filhos através da distribuição de métodos anticoncepcionais. Além dessas mudanças que representam muito mais um resgate da proposta de planejamento familiar, é necessário adequar o programa ao modelo de desenvolvimento. A perspectiva desenvolvimentista dentro do planejamento familiar contribuirá para o aumento da qualidade de vida tanto das unidades familiares como também da sociedade.

As hipóteses explicativas para as falhas do atual modelo de planejamento familiar são necessárias para a compreensão da importância das mudanças sugeridas. Entretanto, estudos subsequentes precisam ser realizados para testar a eficácia de tais argumentos. Os resultados deste estudo serão utilizados para desenvolver um modelo de planejamento familiar adaptado às necessidades regionais presentes nas áreas rurais da região do Recôncavo da Bahia.

As mudanças propostas não podem ser caracterizadas como simples, tampouco fáceis de serem implementadas. Entretanto, parecem bastante atrativas para consubstanciar as mudanças sociais esperadas pelo planejamento familiar. As classes sociais mais baixas precisam de recursos socioeducativos aplicados com metodologias adequadas a essas populações.

\section{Colaboradores}

JC Santos trabalhou na concepção, no delineamento, análise e interpretação dos dados e sua revisão crítica; PM Freitas trabalhou na concepção, no delineamento, análise e interpretação dos dados e redação do artigo.

\section{Agradecimentos}

À Coordenação de Aperfeiçoamento de Pessoal de Nível Superior (Capes), à Fundação de Amparo à Pesquisa do Estado da Bahia (Fapesb) e ao Conselho Nacional de Desenvolvimento Científico e Tecnológico (CNPq). 


\section{Referências}

1. Carvalho JAM, Brito F. A demografia brasileira e o declínio da fecundidade no Brasil: contribuições, equívocos e silêncios. Revista Brasileira Estado e População 2005; 22(2):351-369.

2. Moura ERF, Silva RM. Informação e planejamento familiar como medidas de promoção da saúde. Cien Saude Colet 2004; 9(4):1023-1032.

3. Moura ERF, Silva RM, Galvão MTG. Dinâmica do atendimento em planejamento familiar no Programa Saúde da Família no Brasil. Cad Saude Publica 2007; 23(4):961-970.

4. Brasil. Ministério da Saúde. Agenda Nacional de Prioridades de Pesquisa em Saúde. Brasília: Ministério da Saúde; 2004. [acessado 2006 nov]. Disponível em: www.saude.gov.br

5. Minuchin S. The seductions of constructivism. $\mathrm{Fa}$ mily Therapy Networker 1991; 15:47-50.

6. Fernandes MFM. Mulher, família e reprodução: um estudo de caso sobre o planejamento familiar em periferia do Recife, Pernambuco, Brasil. Cad Saude Publica 2003; 19(Supl.2):S253-S261.

7. Osis MJD, Faúndes A, Makuch MY, Mello MB, Sousa MH, Araújo MJO. Atenção ao planejamento familiar no Brasil hoje: reflexões sobre os resultados de uma pesquisa. Cad Saude Publica 2006; 22(11):2481-2490.

8. Bruce J. Fundamental elements of the quality of care: a simple framework. Studies Family Planning 1990; 21(2):61-91.

9. Arruda BKG. Um novo enfoque da educação médica. Revista Brasileira de Saúde Materno-Infantil 2002; 2(2):89-90.

10. Camiá GEK, Marin HF, Barbieri M. Diagnósticos de enfermagem em mulheres que frequentam serviço de planejamento familiar. Revista Latino-Americana de Enfermagem 2001; 9(2):26-34.

11. Fonseca-Sobrinho D. Estado e população: uma história do planejamento familiar no Brasil. Rio de Janeiro: Rosa dos Tempos; 1993.

12. Osis MJD. PAISM: um marco na abordagem da saúde reprodutiva no Brasil. Cad Saude Publica 1998; 14(Supl.1):25-32.

13. Costa AM. Planejamento familiar no Brasil. [acessado 2002 dez 6]. Disponível em: http://www.cfm.org. br/revista/bio2v4/ planeja.html

14. Lam D, Marteleto L. A dinâmica da escolaridade das crianças brasileiras durante a transição demográfica: aumento no tamanho da coorte versus diminuição no tamanho da família. Belo Horizonte: UFMG/Cedeplar; 2004.

15. Carvalho MLO, Pirotta KCM, Schor N. Participação masculina na contracepção pela ótica feminina. Rev Saude Publica 2001; 35(1):23-33.
16. Sen A. Desenvolvimento como liberdade. São Paulo: Companhia das Letras; 2000.

17. Osis MJD, Hardy E, Faúndes A, Alves G, Balarezo G. Opinião das mulheres sobre as circunstâncias em que os hospitais deveriam fazer abortos. Cad Saude Publica 1994; 10(3):320-330.

18. Pekrun R. Familie, Schule und Entwicklung. In: Walper S, Pekrun R, organizadores. Familie und Entwicklung: Aktuelle Perspektiven der Familienpsychologie. Göttingen: Hogrefe; 2001.

19. Green RG, Harris RN, Forte JA, Robinson M. Evaluating FACES III and the Circumplex Model: 2.440 families. Family Process 1991; 30:55-73.

20. Duval E. Marriage and family development. Philadelphia: Lippincott; 1977.

21. Dessen MA, Braz MP. A família e suas inter-relações com o desenvolvimento humano. In: Dessen MA, Costa Júnior AL, organizadores. A ciência do desenvolvimento humano: tendências atuais $e$ perspectivas futuras. Porto Alegre: Artmed; 2005. p. 113-131.

22. Schwartz RC. Terapia dos sistemas familiares. São Paulo: Roca; 2004

23. Hines PM. O ciclo de vida familiar nas famílias negras pobres. In: Carter B, McGoldrick M, organizadores. As mudanças no ciclo de vida familiar: uma estrutura para a terapia familiar. Porto Alegre: Artes Médicas; 1995. p. 440-467.

24. Carter B, McGoldrick M. As mudanças no ciclo de vida familiar: uma estrutura para a terapia familiar. Porto Alegre: Artes Médicas; 1995.

25. Olson DH, Russel CS, Sprenkle DH. Circumplex Model of Marital and Family Systems: IV. Theoretical Update. Family Process 1983; 22:69-83.

26. Minuchin S. Families and family therapy. Cambridge: Harvard University Press; 1974.

27. Pinto IA. Planejamento familiar: generalidades, aspectos demográficos, políticos e sociais. In: Halbe H, organizador. Tratado de ginecologia. São Paulo: Editora Roca; 1987. v. 1. p. 309-407.

Artigo apresentado em 12/05/2008

Aprovado em 19/01/2009

Versão final apresentada em 06/02/2009 